\title{
Intracranial hemorrhage induced uncontrolled seizure in a deceased donor liver transplant patient
} -a case report-

\author{
Seung-Young $\mathrm{Oh}^{1}$, Hannah Lee ${ }^{2}$, Yang-Hyo Park ${ }^{2}$, and Ho Geol Ryu ${ }^{2}$ \\ Departments of ${ }^{1}$ Surgery, ${ }^{2}$ Anesthesiology and Pain Medicine, Seoul National University Hospital, Seoul, Korea
}

Seizure is the second most common neurologic complication after liver transplantation and may be caused by metabolic abnormalities, electrolyte imbalance, infection, and immunosuppressant toxicity. A 61-year-old male patient underwent liver transplantation due to hepatitis B virus-related liver cirrhosis with portal systemic encephalopathy. The immediate postoperative course of the patient was uncomplicated. However, on postoperative day (POD) 6, weakness developed in both lower extremities. No abnormal findings were detected on a brain computed tomography (CT) scan on POD 8 , but a generalized tonic clonic seizure developed which was difficult to control even with multiple antiepileptic drugs. A follow-up brain CT scan on POD 15 showed a $2.7 \mathrm{~cm}$ sized acute intracranial hemorrhage (ICH) in the left parietal lobe. The patient's mental status improved after 2 months and he was able to communicate through eye blinking or head shaking. Our case reports an acute ICH that manifested into a refractory seizure in a patient who underwent a liver transplant.

Key Words: Encephalopathy, Intracranial hemorrhages, Liver transplantation, Seizures.

Neurologic complications after liver transplantation are more common compared to those after other solid organ transplantations, ranging from $8 \%$ to $71 \%$. Seizure is the second most common neurologic complication after liver transplantation, ranging from $3 \%$ to $20 \%$ [1-3]. Common causes of seizures in liver transplant recipients are metabolic abnormalities, electrolyte

Corresponding author: Ho Geol Ryu, M.D., Ph.D.

Department of Anesthesiology and Pain Medicine, Seoul National University Hospital, 101, Daehak-ro, Jongno-gu, Seoul 03080, Korea

Tel: 82-2-2072-2467, Fax: 82-2-747-5639

E-mail: hogeol@gmail.com

ORCID: http://orcid.org/0000-0001-8952-6049

Received: June 18, 2015.

Revised: October 27, 2015.

Accepted: November 17, 2015.

Korean J Anesthesiol 2016 October 69(5): 527-531 http://dx.doi.org/10.4097/kjae.2016.69.5.527 imbalance, infection, and immunosuppressant toxicity [4-6].

We experienced a case in which a patient showed repetitive generalized tonic clonic seizures (GTCS) after liver transplantation without any evidence of a structural brain lesion. The patient was eventually diagnosed with intracranial hemorrhage (ICH) which was thought to be the start point for repetitive GTCS.

\section{Case Report}

A 61-year-old male patient was admitted to manage hepatitis B virus-related liver cirrhosis. He was diagnosed with portal vein thrombosis and portal systemic encephalopathy for which he received supportive care with lactulose.

The patient underwent donor liver transplantation and was admitted to the surgical intensive care unit for postoperative care. Medications included aspirin and nafamostat mesilate to prevent thrombosis, prostaglandin E1 to vasodilate the anasto-

(c) This is an open-access article distributed under the terms of the Creative Commons Attribution Non-Commercial License (http://creativecommons.org/ licenses/by-nc/4.0/), which permits unrestricted non-commercial use, distribution, and reproduction in any medium, provided the original work is properly cited. 
mosed vessels, tacrolimus and prednisolone for immunosuppression, and ampicillin/sulbactam, fluconazole, and nystatin for prophylaxis against infection.

The patient showed deteriorated mental status and decreased motor power on both upper extremities (grade III) and lower extremities (grade I) on postoperative day (POD) 6. Lower extremity motor power improved (from grade I to III) on POD 7. A neurologic examination conducted by a neurologist. The Glasgow Coma Score was $12(4 / 2 / 6)$, and the pupil reflex was prompt. The patient developed GTCS on POD 8, with a deviated eye ball (3 o'clock direction) for 2 min, which stopped after administering lorazepam. Levetiracetam (2,000 mg/day) and pregabalin (150 mg/day) were administered to control the seizure. However, seizures with abnormal mouth chewing movements tended to be triggered when the patient's position was changed or during pharyngeal suction. A brain computed tomography (CT) scan (Fig. 1A) failed to show any evidence of stroke. Over the next 6 days (PODs 9-15), the pregabalin dose was increased

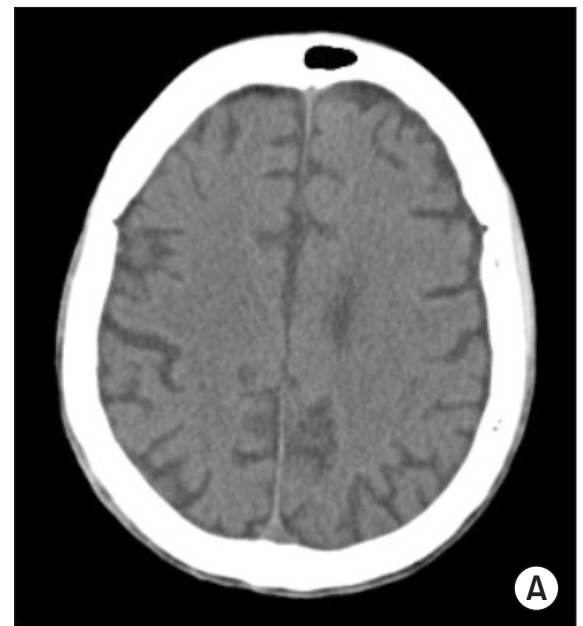

POD 9

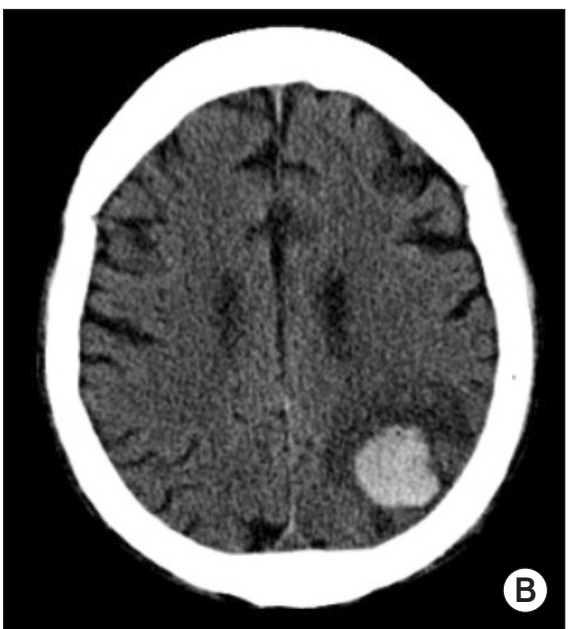

POD 15

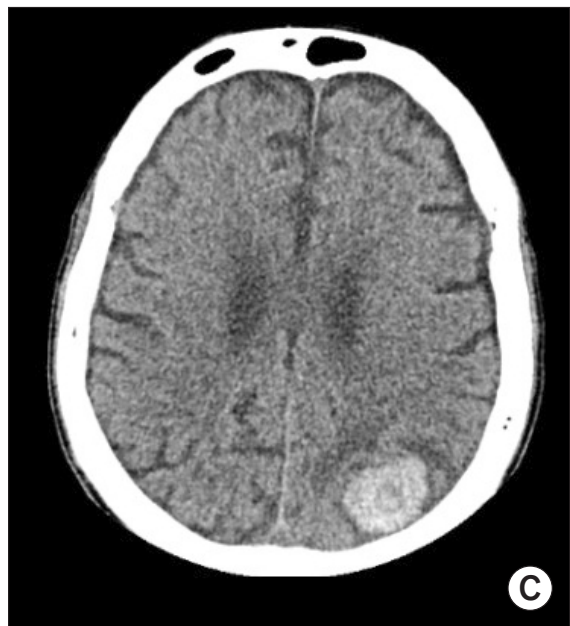

POD 16

Fig. 1. CT findings are demonstrated. (A) There is no evidence of acute intracranial hemorrhage or infarct. And there is no abnormal enhancing lesions or other focal lesions in the brain parenchyma. (B) Acute intracranial hemorrhage (ICH) sized $2.7 \mathrm{~cm}$ in left parietal lobe showed high attenuation. (C) The size of high attenuated ICH was slightly decreased. POD: postoperative day.

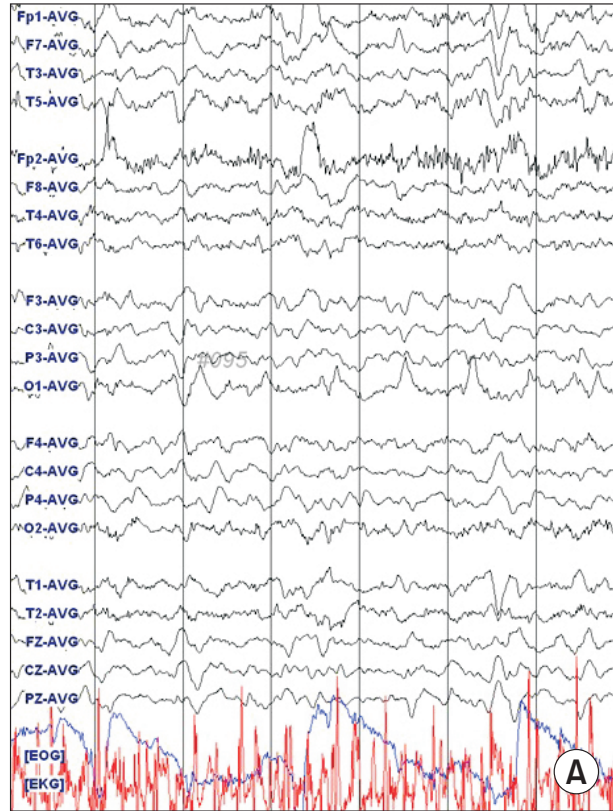

Ictal EEG

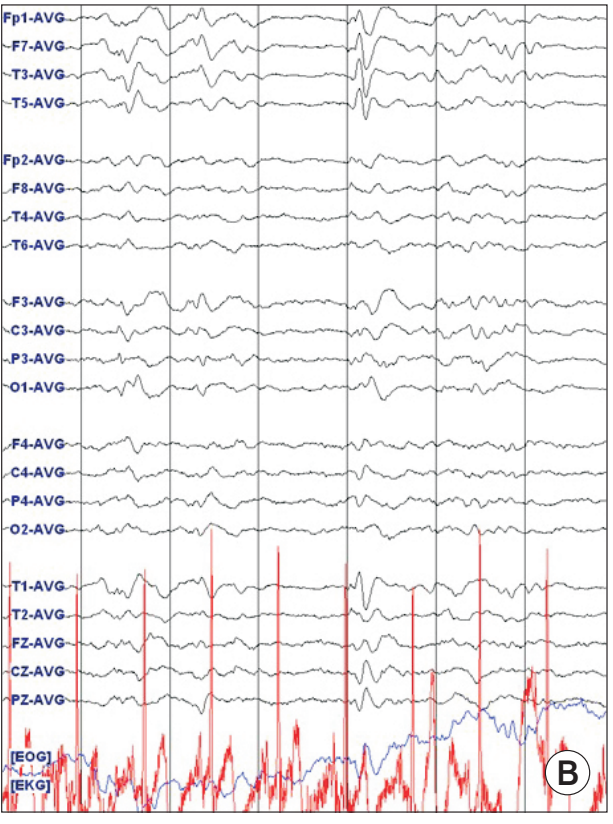

Interictal EEG
Fig. 2. (A) Ictal electroencephalography (EEG) showed left hemisphere dominant waves. (B) Interictal EEG showed slow waves with low voltage over the left lead, especially parietal lobe. 
to $300 \mathrm{mg} /$ day, and fosphenytoin (400 mg/day) was added. A subsequent brain CT (POD 15) scan revealed a $2.7 \mathrm{~cm}$ sized acute ICH in the left parietal lobe (Fig. 1B). Electroencephalography (EEG) showed left hemisphere dominant waves during the ictal state and slow waves with low voltage over the left lead, particularly the parietal lobe, during the interictal state (Fig. 2). Aspirin, which is prescribed routinely after liver transplantation, was stopped. A follow-up brain CT scan taken 2 days later showed that the existing ICH decreased slightly in sized compare to that on previous CT scan, and there was no evidence of a new lesion (Fig. 1C). After increasing the dose of antiepileptic drugs (AED), adding another AED (400 mg/day lacosamide), and administering thiopental (100 $\mathrm{mg}$ ) for breakthrough seizures, the frequency of seizures decreased slowly, and the EEG improved over time.

Brain magnetic resonance imaging (MRI) was performed 3 months after liver transplantation, and the ICH had decreased by $50 \%$ (Fig. 3). The patient was discharged 2 weeks after the MRI and was able to blink his eyes and shake his head.

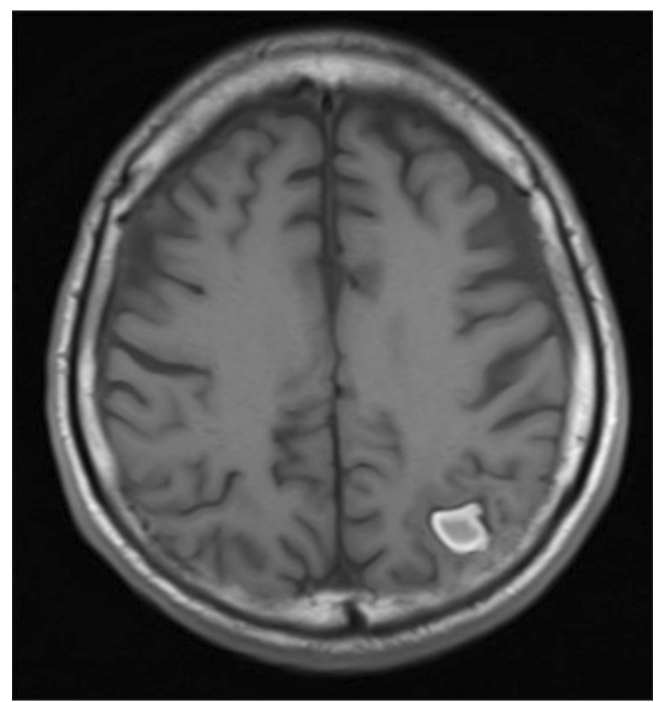

Fig. 3. Magnetic resonance imaging findings demonstrated that the size if the intracranial hemorrhage was reduced to half.

\section{Discussion}

As the definitive cure for end-stage liver disease, liver transplantation has become continuously more frequent over the past few decades. Postoperative complications and mortality have decreased with the improvements in surgical techniques and postoperative management, and survivors can enjoy an excellent quality of life $[7,8]$. However, the risk of complications remains high and critical complications can result in failure of the transplanted liver. Thus, caution and preventive measures are required.

Neurologic complications after liver transplantation are relatively rare with a reported rate of $1.5-4.7 \%$. The most common neurologic complication is encephalopathy followed by seizure and $\mathrm{ICH}[1,3,9]$. However, the mortality rate in patients with $\mathrm{ICH}$ after liver transplantation is $>65 \%[2,10,11]$. Therefore early detection and adequate management are critical.

The risk of bleeding in liver transplant recipients is relatively high for several reasons. First, the transplanted liver becomes fully functional and regains adequate coagulation status after some time. Second, anti-platelet therapy is routinely used to prevent vascular thrombosis. Blood products including coagulation factors are avoided for the same reason and to evaluate recovery of the transplanted liver. Third, relative thrombocytopenia is tolerated to avoid thrombotic complications, which can be devastating. Considering our patient's coagulation profile (Table 1), the cause of ICH may have been the anti-platelet medication and postoperative coagulopathy due to post-transplantation liver dysfunction.

However, the interactions between AEDs and immunosuppressants and a possible change in activity of the AEDs due to altered serum protein levels have made newer AEDs preferred for use in liver transplant recipients. Moreover, our patient was drowsy before the liver transplantation. The laboratory findings of our patient, including the coagulation profile (Table 1) arterial blood gases, and electrolyte, glucose (Table 2), and hemoglobin levels were within normal ranges, and the initial brain CT scan after the first seizure showed no structural abnormalities. There was slight hyponatremia but the initial sodium level was slightly low, and no dramatic changes in sodium level occurred. Thus, we presumed that an electrolyte imbalance was probably not the

Table 1. Changes in Coagulation Profile

\begin{tabular}{|c|c|c|c|c|c|c|c|c|}
\hline & Post-reperfusion & POD 0 & POD 1 & POD 3 & POD 6 & POD 8 & POD 9 & POD 15 \\
\hline PT (INR) & 2.06 & 1.43 & 1.33 & 1.06 & 0.98 & 0.96 & 0.97 & 1.15 \\
\hline aPTT (sec) & 64.9 & 45.3 & 39.9 & 32.0 & 25.9 & 24.8 & 24.2 & 32.7 \\
\hline Platelet $\left(\times 10^{3} / \mathrm{ul}\right)$ & 52 & 74 & 30 & 57 & 47 & 45 & 67 & 137 \\
\hline
\end{tabular}

POD: postoperative day. 
Table 2. Postoperative Laboratory Findings

\begin{tabular}{|c|c|c|c|c|c|c|}
\hline & POD 1 & POD 3 & POD 6 & POD 8 & POD 9 & POD 15 \\
\hline $\mathrm{pH}$ & 7.44 & 7.46 & 7.48 & 7.455 & 7.464 & 7.441 \\
\hline $\mathrm{Na}(\mathrm{mmol} / \mathrm{L})$ & 133 & 135 & 135 & 132 & 130 & 131 \\
\hline $\mathrm{K}(\mathrm{mmol} / \mathrm{L})$ & 4.0 & 4.3 & 3.4 & 4.6 & 4.8 & 4.8 \\
\hline Glucose (mg/dl) & 156 & 167 & 130 & 157 & 114 & 207 \\
\hline Lactic acid $(\mathrm{mmol} / \mathrm{L})$ & 2.1 & & 0.5 & & 0.6 & 1.0 \\
\hline
\end{tabular}

POD: postoperative day.

main cause for the seizures.

Because ICH was low on the differential diagnosis list, aspirin (100 mg/day) was continued for 6 days after the seizure, which may have accelerated the progression of ICH. A better patient outcome may have been anticipated had we detected the ICH sooner and adjusted or stopped the antiplatelet agent earlier.

The initial negative brain CT scan can be explained by the timing of the scan. Considering the final size of the hemorrhage and its slow rate of progression, the initial hemorrhage may have been too small to detect by the initial brain CT scan. Accuracy of the first head CT scan within 24 hours of an acute is about $70 \%$, with $61.9 \%$ sensitivity and $71.4 \%$ specificity [12]. MRI has advantages of enabling diagnosis of an ischemic lesion and not exposing the patient to ionizing radiation $[13,14]$. Mullins et al. reported the superiority of diffusion-weighted MRI compared with that of CT in patients suffering an acute (within 24 hours) stroke with unknown onset of neurologic symptoms, particularly when performed within 6 hours. We could not determine the exact onset time of the neurologic symptoms because the mental status of our patient was not fully recovered; therefore, diffusion-weighted MRI may have been a better choice for the initial evaluation. However the long duration of MRI limits practical use, particularly in patients with strong suspicion of bleeding.

The EEG findings suggested that the focus of the seizure was the left parietal lobe, which correlated with the ICH location. The CT scan showed no evidence of high intracranial pressure (such as midline shift or loss of sulci), so the degree hemorrhaging did not meet the indication for surgical treatment. However, interactions between AEDs and immunosuppressants used for liver transplantation and the altered serum protein levels that can affect the activity of AEDs makes newer AEDs preferred for liver transplant recipients $[4,6]$. Levetiracetam, pregabalin, and lacosamide were selected because of their minimal drug interactions, and fosphenytoin was added for repetitive seizure control in this patient.

In our case, effective communication was impaired because of the underlying hepatic encephalopathy and the long duration mechanical ventilation. Although ICH is rare, considering that liver transplant recipients have high risk of bleeding, the presence of ICH should be considered when neurologic symptoms develop or when mental recovery is slow.

\section{References}

1. Menegaux F, Keeffe EB, Andrews BT, Egawa H, Monge H, Concepcion W, et al. Neurological complications of liver transplantation in adult versus pediatric patients. Transplantation 1994; 58: 447-50.

2. Bronster DJ, Emre S, Boccagni P, Sheiner PA, Schwartz ME, Miller CM. Central nervous system complications in liver transplant recipients-incidence, timing, and long-term follow-up. Clin Transplant 2000; 14: 1-7.

3. Ghaus N, Bohlega S, Rezeig M. Neurological complications in liver transplantation. J Neurol 2001; 248: 1042-8.

4. Chabolla DR, Wszolek ZK. Pharmacologic management of seizures in organ transplant. Neurology 2006; 67(12 Suppl 4): S34-8.

5. Mirski MA1, Varelas PN. Seizures and status epilepticus in the critically ill. Crit Care Clin 2008; 24: 115-47, ix.

6. Shepard PW, St Louis EK. Seizure treatment in transplant patients. Curr Treat Options Neurol 2012; 14: 332-47.

7. Tanikella R, Kawut SM, Brown RS Jr, Krowka MJ, Reinen J, Dinasarapu CR, et al. Health-related quality of life and survival in liver transplant candidates. Liver Transpl 2010; 16: 238-45.

8. Kuramitsu K, Fukumoto T, Iwasaki T, Tominaga M, Matsumoto I, Ajiki T, et al. Long-term complications after liver transplantation. Transplant Proc 2014; 46: 797-803.

9. Ardizzone G, Arrigo A, Schellino MM, Stratta C, Valzan S, Skurzak S, et al. Neurological complications of liver cirrhosis and orthotopic liver transplant. Transplant Proc 2006; 38: 789-92.

10. Ling L, He X, Zeng J, Liang Z. In-hospital cerebrovascular complications following orthotopic liver transplantation: a retrospective study. BMC Neurol 2008; 8: 52.

11. Moreno E, Gómez SR, Gonzalez I, Loinaz C, Garcia I, Perez A, et al. Neurologic complications in liver transplantation. Acta Neurol Scand 
1993; 87: 25-31.

12. Mullins ME, Schaefer PW, Sorensen AG, Halpern EF, Ay H, He J, et al. CT and conventional and diffusion-weighted MR imaging in acute stroke: study in 691 patients at presentation to the emergency department. Radiology 2002; 224: 353-60.

13. Wong GK, Siu DY, Ahuja AT, King AD, Yu SC, Zhu XL, et al. Comparisons of DSA and MR angiography with digital subtraction angiography in 151 patients with subacute spontaneous intracerebral hemorrhage. J Clin Neurosci 2010; 17: 601-5.

14. Wong GK, Siu DY, Abrigo JM, Ahuja AT, Poon WS. Computed tomographic angiography for patients with acute spontaneous intracerebral hemorrhage. J Clin Neurosci 2012; 19: 498-500. 